\title{
A bulk modulus dependent linear model for acoustical imaging
}

\author{
Jean Martial Mari ${ }^{\mathrm{a})}$ \\ Department of Bioengineering, Royal School of Mines, Imperial College London, South Kensington \\ Campus, London SW7 2BP, United Kingdom \\ Thierry Blu \\ Department of Electronic Engineering, Chinese University of Hong Kong, Shatin New Territories, \\ Hong Kong \\ Olivier Bou Matar \\ Laboratoire Européen Associé en MagnétoAcoustique de la Matière Condensée (IEMN-DOAE UMR CNRS \\ 8520), Ecole Centrale de Lille, BP 48, 59651 Villeneuve d'Ascq Cedex, France \\ Michael Unser \\ Biomedical Imaging Group, EPFL/STI/IMT/LIB, CH-1015 Lausanne VD, Switzerland \\ Christian Cachard \\ CREATIS, Université de Lyon, INSA-Lyon, Université Lyon 1, CNRS UMR5220, INSERM U630 Bâtiment \\ Blaise Pascal, INSA, 7 Avenue Jean Capelle, F69621 Villeurbanne Cedex, France
}

(Received 26 February 2007; revised 24 September 2008; accepted 3 February 2009)

\begin{abstract}
Modeling the acoustical process of soft biological tissue imaging and understanding the consequences of the approximations required by such modeling are key steps for accurately simulating ultrasonic scanning as well as estimating the scattering coefficient of the imaged matter. In this document, a linear solution to the inhomogeneous ultrasonic wave equation is proposed. The classical assumptions required for linearization are applied; however, no approximation is made in the mathematical development regarding density and speed of sound. This leads to an expression of the scattering term that establishes a correspondence between the signal measured by an ultrasound transducer and an intrinsic mechanical property of the imaged tissues. This expression shows that considering the scattering as a function of small variations in the density and speed of sound around their mean values along with classical assumptions in this domain is equivalent to associating the acoustical acquisition with a measure of the relative longitudinal bulk modulus. Comparison of the model proposed to Jensen's earlier model shows that it is also appropriate to perform accurate simulations of the acoustical imaging process.
\end{abstract}

(C) 2009 Acoustical Society of America. [DOI: 10.1121/1.3087427]

PACS number(s): 43.80.Cs, 43.20.Bi, 43.35.Bf, 43.80.Vj [LLT] Pages: 2413-2419

\section{INTRODUCTION}

Ultrasonic imaging is a complex process during which a focused beam is transmitted through a soft biological tissue and scattered by inhomogeneities stemming from local variations in the intrinsic mechanical properties. Ideally, knowing the electromechanical transfer function of the probe and the measured backscattered signal, called the radiofrequency (RF) signal, should solve the inverse problem, thus retrieving the real scattering profiles. This consists in removing the influence of the acquisition tool and displaying an ultrasonic image of higher quality. Unfortunately, the problems that arise from the complexity of the scattering process ${ }^{1-6}$ in the tissues and from the technical limits of the ultrasonic probes render the mathematical inversion an open problem. To be solved, the problem has to be regularized by formulating assumptions on the propagating ultrasound wave, the behavior of the continuous collection of scatterers, and finally the range of variation of the local mechanical properties. Among

${ }^{a)}$ Electronic mail: jm.mari@imperial.ac.uk the possible approaches and the hypothesis these approaches imply, one solution consists in searching for a linear description of the problem, ${ }^{7-9}$ transforming the inversion step into a well-known deconvolution process. ${ }^{10}$ A linear description means that the response of a collection of scatterers is the sum of the responses of each constituent scatterer. This form then makes it possible to quantitatively solve the inverse problem, while the mathematical expression of the problem can reveal the dependence of the estimated scattering coefficient on a particular intrinsic mechanical parameter. Specifically, this sheds light on the consequences of the assumptions on the nature of the resulting scattering coefficient and also reveals a posteriori the approximations required, as a cost for obtaining a simplified description of the acoustical process.

Linear descriptions for the ultrasonic imaging process of soft tissues are well known and have been extensively developed in literature, such as in Refs. 1, 2, 7, and 8. As previously shown, one of their main results consists in expressing the scattering coefficient as a function of the local intrinsic properties of the medium. In the past, Gore and Leeman ${ }^{1}$ and 
more recently Zemp et $a .^{7}$ showed that under various assumptions in the field and the insonified medium, the scattering coefficient depends on the adiabatic compressibility coefficient and density. The approach of Zemp et al. ${ }^{7}$ contributes an original solution to space-varying point spread functions (PSFs) and allows for a statistical approach to the problem. Fatemi and $\mathrm{Kak}^{2}$ expressed the scattering coefficient as a function of sound speed only, though strong hypotheses were imposed. Jensen ${ }^{8}$ derived the scattering coefficient as a function of sound speed and density. This model is very interesting because it provides an expression of the model where each part can be measured or defined, so that one can, respectively, partly retrieve the scatterer distribution or simulate medical ultrasound imaging. Unfortunately, the first-order expression of Jensen's scattering coefficient ${ }^{8}$ does not take advantage of the stronger hypothesis involved and the final expression contains unused approximations.

In this paper, it is shown that those expressions of the ultrasonic process and their scattering coefficients, a function of sound speed and density, can be linked to the variations in the sole longitudinal bulk modulus. As a result, the ultrasound scanning process may be interpreted under the classical assumptions as the measurement of a single parameterrelated to the medium's properties-rather than a local reflectivity combining several parameters that is noninvertible. Fatemi and $\mathrm{Kak}^{2}$ calculated this coefficient as a function of sound speed, though this was done by ignoring local density variation, which is an unreasonable assumption in medical imaging. We show that their coefficient can be interpreted as an approximation of a more general expression.

The expansion and solution of the wave equation proposed are very close to Jensen's approach; ${ }^{8}$ consequently the results that have already been demonstrated in detail are used directly, and our contribution is highlighted. Jensen's development ${ }^{8}$ was chosen because it offers flexible and widespread solutions for the simulation of ultrasonic imaging with various probe geometries, and, like the model of Zemp et al., ${ }^{7}$ it can also take into account both attenuation ${ }^{11}$ and space-varying PSFs.

The paper is organized as follows: First, the general wave equation is modified to establish the expression to be solved under the assumptions chosen. An expression of the received signal is established in Sec. III and the results are discussed in Sec. IV.

\section{DERIVATION OF THE WAVE EQUATION}

Assuming that the particle velocity in the medium is small, which is usually the case at the power dissipated by medical ultrasound scanners, the wave equation can be written as ${ }^{8}$

$$
\nabla^{2} p_{1}(\boldsymbol{r}, t)-\frac{1}{c(\boldsymbol{r})^{2}} \cdot \frac{\partial^{2} p_{1}(\boldsymbol{r}, t)}{\partial t^{2}}=\frac{1}{\rho(\boldsymbol{r})} \nabla \rho(\boldsymbol{r}) \cdot \nabla p_{1}(\boldsymbol{r}, t),
$$

where $p_{1}$ is the pressure perturbation due to the propagation, at location $\boldsymbol{r}$ (Fig. 1) and time $t, \rho$ is the local density, and $c$ is the local speed of sound. Bold characters stand for threedimensional triplets. $\boldsymbol{\nabla}$ is the gradient operator and $\nabla^{2}$ is the

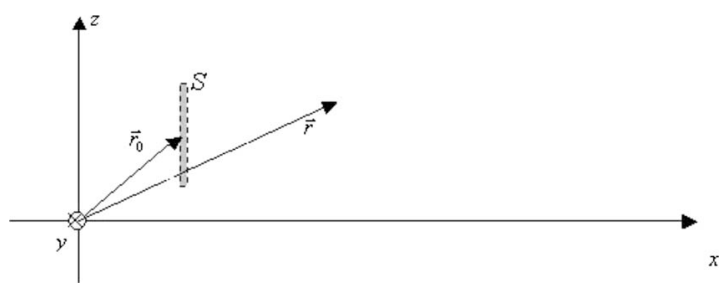

FIG. 1. Definition of the transducer's surface $(S)$, position $\left(r_{0}\right)$, and the transmission/scattering location $\boldsymbol{r}$.

Laplace operator. The solution method consists in isolating all the terms expressing a propagating wave at a constant speed on the left-hand side of the equation, and keeping the others on the right-hand side, which stands for the scattering operator. Since it assumes that the particle velocity is small in the insonified medium, this development is only suitable for linear imaging and does not take into account harmonic tissue imaging ${ }^{12,13}$ and contrast agent imaging. ${ }^{14-17}$ In the later developments, when no ambiguity is possible, the function's variables will be omitted for purposes of legibility.

Reorganizing the left- and right-hand terms of Eq. (1) gives

$$
\underset{c_{0}}{\exists} p_{1}=\operatorname{Fop}\left[p_{1}\right]
$$

with

$$
\underset{c_{0}}{\Xi}=\nabla^{2}(\cdot)-\frac{1}{c_{0}^{2}} \cdot \frac{\partial^{2}}{\partial t^{2}}(\cdot)
$$

the D'Alembert operator in $c_{0}$ and

$$
\text { Fop }=\frac{c^{2}}{c_{0}^{2}} \cdot \frac{\boldsymbol{\nabla} \rho}{\rho} \cdot \nabla(\cdot)-\left(\frac{c^{2}}{c_{0}^{2}}-1\right) \cdot \operatorname{div}(\boldsymbol{\nabla}(\cdot))
$$

the scattering operator. The ultrasonic process is described as the propagation of a progressive wave [left member of Eq. (2)] at constant speed $c_{0}$, which is perturbed by local inhomogeneities (described in the right member). However, contrary to Ref. 8, no first-order expression is made of $c(\boldsymbol{r})$ and $\rho(\boldsymbol{r})$ for the elaboration of the scattering operator Fop, given in Eq. (4), even if it will be developed considering small perturbations. Equation (2) is the equation that has to be solved in order to calculate the expression of the received signal.

\section{CALCULATION OF THE RECEIVED SIGNAL}

The solution is obtained by assuming that the incident field is a solution of the homogeneous part of Eq. (2) and its expression is established following Jensen's method. ${ }^{8}$ Then the scattered field is calculated under the first-order Born approximation, and its integration over the transducer surface finally gives the expression of the received signal, which is a function of the local scattering coefficient.

\section{A. Calculation of the incident field}

The incident field propagating in tissues is assumed to be an eigensolution of the homogeneous part of Eq. (2) and is assumed to derive from a velocity potential $\psi$ in Eq. (5). It is implicitly expressed by Eq. (6) as follows: 


$$
\begin{aligned}
& \boldsymbol{v}(\boldsymbol{r}, t)=\boldsymbol{\psi} \psi(\boldsymbol{r}, t), \\
& \boldsymbol{\nabla} p_{i}(\boldsymbol{r}, t)=-\rho \cdot \frac{\partial \boldsymbol{v}(\boldsymbol{r}, t)}{\partial t} .
\end{aligned}
$$

Combining Eqs. (5) and (6) gives

$$
\boldsymbol{\nabla} p_{i}=-\rho \cdot \frac{\partial \boldsymbol{\nabla} \psi}{\partial t}
$$

where $\psi\left(\boldsymbol{r}, \boldsymbol{r}_{0}, t\right)$ is given at $\boldsymbol{r}$ for a single transducer in $\boldsymbol{r}_{0}$ by the following expression: ${ }^{2}$

$$
\psi\left(\boldsymbol{r}, \boldsymbol{r}_{0}, t\right)=v_{n}(t) * h\left(\boldsymbol{r}, \boldsymbol{r}_{0}, t\right),
$$

where $*$ denotes time convolution, and

$$
h\left(\boldsymbol{r}, \boldsymbol{r}_{0}, t\right)=-\int_{\boldsymbol{q} \in S} \frac{\delta\left(t-\frac{\left|\boldsymbol{r}-\boldsymbol{r}_{0}+\boldsymbol{q}\right|}{c_{0}}\right)}{4 \cdot \pi \cdot\left|\boldsymbol{r}-\boldsymbol{r}_{0}+\boldsymbol{q}\right|} \cdot d^{2} \boldsymbol{q},
$$

where $v_{n}(t)$ is the normal speed of the transducer's surface $S$, and $h\left(\boldsymbol{r}, \boldsymbol{r}_{0}, t\right)$ is the PSF in transmission for a transducer element placed in $\boldsymbol{r}_{0}$.

Then, Eq. (7) gives

$$
\begin{aligned}
\boldsymbol{\nabla} p_{i}\left(\boldsymbol{r}, \boldsymbol{r}_{0}, t\right) & =-\rho \cdot \frac{\partial \boldsymbol{\nabla} \psi\left(\boldsymbol{r}, \boldsymbol{r}_{0}, t\right)}{\partial t} \\
& =-\rho \cdot \frac{\partial v_{n}(t)}{\partial t} * \boldsymbol{\nabla} h\left(\boldsymbol{r}, \boldsymbol{r}_{0}, t\right) .
\end{aligned}
$$

The hypothesis of constant sound speed has been made for propagation (i.e., $c=c_{0}$ ), and we have assumed that $v_{n}(t)$ is constant over the transducer's surface. Equation (10) does not directly provide an expression of the incident field $p_{i}$, but this implicit expression is sufficient for the expression of the received signal. The use of $\rho$ in this equation, and not $\rho_{0}$ as in Ref. 8, is discussed in Sec. IV.

\section{B. Calculation of the scattered field}

The pressure at $\boldsymbol{r}$ is calculated by integrating the ambient field $p_{1}$ over the volume space $V$. This is done using Green's function $^{18}$ and leads to the scattered field ${ }^{8}$

$$
p_{s}\left(\boldsymbol{r}, \boldsymbol{r}_{0}, t\right)=\int_{V} \int_{T} F o p\left[p_{1}\left(\boldsymbol{\xi}, \boldsymbol{r}_{0}, \tau\right)\right] \cdot G(\boldsymbol{\xi}, \tau \mid \boldsymbol{r}, t) \cdot d \tau \cdot d^{3} \boldsymbol{\xi},
$$

where integration is through space $(\xi \in V)$ and time $(\tau \in T)$, and $G$ is Green's function of the homogeneous equation [Eq. (2)]

$$
G(\boldsymbol{\xi}, \tau \mid \boldsymbol{r}, t)=-\frac{\delta\left(t-\tau-\frac{|\boldsymbol{r}-\boldsymbol{\xi}|}{c_{0}}\right)}{4 \cdot \pi \cdot|\boldsymbol{r}-\boldsymbol{\xi}|} .
$$

Under the first-order Born approximation, it is shown ${ }^{8}$ that $p_{1}$ is close to the incident field $p_{i}$, and $p_{s}$ becomes

$$
p_{s}\left(\boldsymbol{r}, \boldsymbol{r}_{0}, t\right)=\int_{V} \int_{T} F o p\left[p_{i}\left(\boldsymbol{\xi}, \boldsymbol{r}_{0}, \tau\right)\right] \cdot G(\boldsymbol{\xi}, \tau \mid \boldsymbol{r}, t) \cdot d \tau \cdot d^{3} \boldsymbol{\xi}
$$

$p_{s}$ now has to be integrated over the transducer's surface in order to express the received signal.

\section{Calculation of the received signal}

The received signal is the scattered pressure field $p_{s}$ integrated over the transducer's surface $S$, convolved with the electromechanical impulse response $E_{m}(t)$ of the transducer,

$$
p_{r}\left(\boldsymbol{r}_{0}^{\prime}, \boldsymbol{r}_{0}, t\right)=E_{m}(t) * \int_{S} p_{s}\left(\boldsymbol{r}_{0}^{\prime}+\boldsymbol{\xi}, \boldsymbol{r}_{0}, t\right) d^{2} \boldsymbol{\xi}
$$

for a receiving transducer placed at point $r_{0}^{\prime}$. Then, considering that

$$
\begin{aligned}
p_{s}\left(\boldsymbol{r}, \boldsymbol{r}_{0}, t\right) & =\int_{V} \int_{T} F o p\left[p_{i}\left(\boldsymbol{\xi}, \boldsymbol{r}_{0}, \tau\right)\right] \cdot G(\boldsymbol{\xi}, \tau \mid \boldsymbol{r}, t) \cdot d \tau \cdot d^{3} \boldsymbol{\xi} \\
& =\int_{V} \int_{T} F_{o p}\left[\nabla p_{i}\left(\boldsymbol{\xi}, \boldsymbol{r}_{0}, \tau\right)\right] \cdot G(\boldsymbol{\xi}, \tau \mid \boldsymbol{r}, t) \cdot d \tau \cdot d^{3} \boldsymbol{\xi}
\end{aligned}
$$

with

$$
\mathrm{Fop}_{2}=\frac{c^{2}}{c_{0}^{2}} \cdot \frac{\boldsymbol{\nabla} \rho}{\rho} \cdot(\cdot)-\left(\frac{c^{2}}{c_{0}^{2}}-1\right) \cdot \operatorname{div}(\cdot),
$$

inserting Eqs. (10) and (16) into Eq. (14) leads to

$$
\begin{aligned}
p_{r}\left(\boldsymbol{r}, \boldsymbol{r}_{0}, t\right)= & -\frac{E_{m}(t)}{4} * \frac{\partial v(t)}{\partial t} * \int_{t} \operatorname{Fop}_{2}\left[\rho \cdot \nabla h\left(\boldsymbol{\xi}, \boldsymbol{r}_{0}, t\right)\right] \\
& * h^{\prime}\left(\boldsymbol{r}_{0}, \boldsymbol{\xi}, t\right) \cdot d^{3} \boldsymbol{\xi}
\end{aligned}
$$

where $h^{\prime}\left(r_{0}^{\prime}, \xi, t\right)$ is Green's function integrated over the transducer's surface, that is, the spatial impulse response of the transducer for reception. This expression is equivalent to

$$
\begin{aligned}
p_{r}\left(\boldsymbol{r}, \boldsymbol{r}_{0}, t\right)= & -\frac{E_{m}(t)}{4} * \frac{\partial v(t)}{\partial t} * \int_{V} F_{V}\left[h\left(\boldsymbol{\xi}, \boldsymbol{r}_{0}, t\right)\right] \\
& * h^{\prime}\left(\boldsymbol{r}_{0}, \boldsymbol{\xi}, t\right) \cdot d^{3} \boldsymbol{\xi} \\
& t
\end{aligned}
$$

with

$$
F o p_{3}=\frac{c^{2}}{c_{0}^{2}} \cdot \nabla \rho \cdot \nabla(\cdot)-\left(\frac{c^{2}}{c_{0}^{2}}-1\right) \cdot \operatorname{div}(\rho \cdot \nabla(\cdot)) .
$$

This expression is approximated by assuming that the perturbation operator is, in fact, applied to the full PSF 


$$
\begin{aligned}
\operatorname{pr}\left(\boldsymbol{r}, \boldsymbol{r}_{0}, t\right) & =\frac{E m(t)}{4} * \frac{\partial v(t)}{\partial t} * \int_{t} F_{V}[h] * h^{\prime} \cdot d^{3} \xi \\
& \approx \frac{E m(t)}{4} * \frac{\partial v(t)}{\partial t} * \int_{t} F o p_{3}\left[h * h^{\prime}\right] \cdot d^{3} \xi
\end{aligned}
$$

This approximation and its implications are discussed in Sec. IV.

Then, defining

$$
h_{p e}\left(\boldsymbol{r}, \boldsymbol{r}_{0}, t\right)=h\left(\boldsymbol{r}, \boldsymbol{r}_{0}, t\right) * h^{\prime}\left(\boldsymbol{r}_{0}, \boldsymbol{r}, t\right)
$$

can be written as

$$
\begin{aligned}
\int_{V} F_{0 p_{3}}\left[h_{p e}\right] \cdot d^{3} \xi= & \int_{V}\left[\alpha \cdot \nabla \rho \cdot \nabla h_{p e}\right. \\
& \left.-(\alpha-1) \cdot \operatorname{div}\left(\rho \cdot \nabla h_{p e}\right)\right] \cdot d^{3} \xi
\end{aligned}
$$

where $\alpha(\boldsymbol{r})=c(\boldsymbol{r})^{2} / c_{0}^{2}$.

Then the Green-Ostrogradsky theorem and the properties of the divergence can be written as

$$
\begin{aligned}
\int_{V} \operatorname{div}(u \cdot \boldsymbol{f}) \cdot \operatorname{div} & =\int_{V}(\boldsymbol{\nabla} u \cdot \boldsymbol{f}+u \cdot \operatorname{div}(\boldsymbol{f})) \cdot \operatorname{div} \\
& =\int_{S_{V}} u \cdot \boldsymbol{f} \cdot \boldsymbol{n} \cdot d s
\end{aligned}
$$

with $\boldsymbol{n}$ the outward normal vector to the surface $S_{V}$ of the integration volume $V$. If $u \cdot f$ decreases rapidly, the integral is null for an unbounded space, leading to the relationship

$$
\int_{V} u \cdot \operatorname{div}(\boldsymbol{f}) \cdot \operatorname{div}=-\int_{V} \boldsymbol{\nabla}(u) \cdot \boldsymbol{f} \cdot \operatorname{div} .
$$

Assuming that $h_{p e}$ varies slowly toward infinity and tends to zero, which is the case for ultrasound beams, and using Eq. (25), Eq. (23) becomes

$$
\begin{aligned}
& \int_{V} \operatorname{Fop}_{3}\left[h_{p e}\right] \cdot d^{3} \xi=\int_{V}\left[\alpha \cdot \nabla \rho \cdot \nabla h_{p e}+\nabla(\alpha-1)\right. \\
& \left.\left.\cdot \rho \cdot \nabla h_{p e}\right)\right] \cdot d^{3} \xi \\
& =\int_{V} \nabla(\alpha \cdot \rho) \cdot \nabla h_{p e} \cdot d^{3} \xi \\
& =-\int_{V}(\alpha \cdot \rho) \cdot \nabla^{2} h_{p e} \cdot d^{3} \xi
\end{aligned}
$$

and the final expression for the scattered field is

$$
\begin{aligned}
p_{r}\left(\boldsymbol{r}_{0}, t\right)= & \rho_{0} \cdot \frac{E_{m}(t)}{4} * \frac{\partial v(t)}{\partial t} * \int_{t}\left[\frac{c^{2} \cdot \rho}{c_{0}^{2} \cdot \rho_{0}}\right] \\
& \cdot \nabla^{2} h_{p e}\left(\boldsymbol{\xi}, \boldsymbol{r}_{0}, t\right) \cdot d^{3} \boldsymbol{\xi}
\end{aligned}
$$

$$
\begin{aligned}
\Leftrightarrow p_{r}\left(\boldsymbol{r}_{0}, t\right)= & \rho_{0} \cdot \frac{E_{m}(t)}{4} * \frac{\partial v(t)}{\partial t} * \int_{V} \nabla^{2}\left[\frac{K(\boldsymbol{\xi})}{K_{0}}\right] \\
& \cdot h_{p e}\left(\boldsymbol{\xi}, \boldsymbol{r}_{0}, t\right) \cdot d^{3} \boldsymbol{\xi},
\end{aligned}
$$

where $K=c^{2} \cdot \rho$ is the longitudinal bulk modulus, which is the inverse of the compressibility modulus and in this very case equal to the longitudinal Young modulus, ${ }^{3,4}$ and $K_{0}$ its average value such that

$$
K(\boldsymbol{r})=K_{0}+\Delta K(\boldsymbol{r})
$$

assuming that $h_{p e}$ is the solution of the homogeneous part of Eq. (2). Using Eq. (30) leads to

$$
\begin{aligned}
p_{r}\left(\boldsymbol{r}_{0}, t\right)= & \frac{\rho_{0}}{2 \cdot c_{0}^{2}} \cdot E_{m}(t) * \frac{\partial^{3} v(t)}{\partial t^{3}} * \int_{t} h_{p e}\left(\boldsymbol{\xi}, \boldsymbol{r}_{0}, t\right) \\
& \cdot\left[\frac{K(\boldsymbol{\xi})}{K_{0}}+\varphi\right] \cdot d^{3} \boldsymbol{\xi},
\end{aligned}
$$

where $K / K_{0}+\varphi$ is the scattering coefficient $R$. The term $\varphi$ is a constant that is naturally discarded by the convolution form, since the imaging process is only sensitive to the variations in the local properties. It is introduced so that the scattering coefficient can be extracted from the general equation while retaining a physical meaning, but has no impact on the scattered pressure. As the scattering coefficient itself should be zero when $K=K_{0}$, the value for the constant is $\varphi=-1$, and the complete expression for $R$ is

$$
R=\frac{K-K_{0}}{K_{0}}
$$

Finally, the model expression can be reduced to

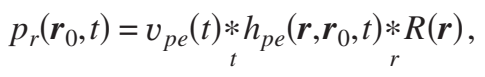

where $*$ denotes spatial convolution and

$$
\begin{aligned}
& v_{p e}(t)=\frac{\rho_{0}}{4 c_{0}^{2}} \cdot E_{m}(t) * \frac{\partial^{3} v(t)}{\partial t^{3}}, \\
& h_{p e}\left(\boldsymbol{r}, \boldsymbol{r}_{0}, t\right)=h\left(\boldsymbol{r}, \boldsymbol{r}_{0}, t\right) * h\left(\boldsymbol{r}_{0}, \boldsymbol{r}, t\right), \\
& R(\boldsymbol{r})=\frac{K(\boldsymbol{r})-K_{0}}{K_{0}}, \\
& K=c^{2} \cdot \rho .
\end{aligned}
$$

The term $R$ in Eq. (37) is the scattering term, which is defined here as the relative radial bulk modulus. This expression is valid only for small variations in the bulk modulus around its average value, that is, with $\Delta K(\boldsymbol{r}) \ll K_{0}$, if $\Delta K(\boldsymbol{r})$ is the variation of $K$ at point $\boldsymbol{r}$. As in the previous model, the speed of sound and density variations cannot be estimated independently; however, under the assumptions, in the scattering coefficient, they form the expression of another local parameter: longitudinal bulk modulus. 


\section{DISCUSSION}

The proposed model is developed under the same main hypotheses as the usual linear descriptions of the ultrasonic imaging process, ${ }^{1,2,7,8}$ essentially linear propagation and the Born approximation. The main difference is the use of nonapproximated forms for $c$ and $\rho$, but other assumptions have been modified or adapted.

\section{A. Expression of the incident field}

The expression of the incident field used in this model is slightly different from Jensen's expression. ${ }^{8}$ We hypothesize that it is possible to consider that the density $\rho$ is fully unknown at the measurement point and thus not "partially" equal to $\rho_{0}$, as implied by Eq. (27) in Ref. 8. Indeed, in Ref. $8, \rho_{0}$ is the density at $\boldsymbol{r}$ where density is assumed to be unknown. The present hypothesis is not newly introduced because once the influence of sound speed and density on the propagation are considered separately, and once a linear behavior of the system is assumed (which means that the response of the scattering coefficient collection is the sum of the responses of each one), there is no need to consider, at a certain point $\boldsymbol{r}$, the past density along the propagation profile, which is implicitly set to $\rho_{0}$. Here the field is assumed to be known until the ultrasound pulse reaches inhomogeneity, where the density and sound speed variations are then fully taken into account. This appears in the model being developed through the use of $\rho$ in Eq. (6). The solution was performed assuming that the propagation medium's density and speed of sound are equal to their average values $\rho_{0}$ and $c_{0}$ everywhere, scattering point $\boldsymbol{r}$ included, but also that these quantities are varying at this point. This contradiction regarding the density changes is moved here by making the same hypothesis until the incidence on a measurement point $\boldsymbol{r}$, but not at this point. The difference is that the pressure changes at point $\boldsymbol{r}$ are more accurately estimated, through a better use of the assumptions required to assume that the incoming pressure is known.

On the other hand, the use of $c_{0}$ in Eqs. (9) and (12) emphasizes that a linear development includes contradictions, because this assumption of propagation at the mean speed of sound includes the estimation point $\boldsymbol{r}$. This last problem cannot be avoided here. A more complete description should imply the local variations in the speed of sound so as to take into account phase changes during propagation, as done very recently in Ref. 19 .

\section{B. Approximation of Equation (21)}

The development proposed is possible by considering that the scattering operator is taken as Eq. (21) on the entire PSF, including the time convolution with the reception profile $h^{\prime}$. This operation expresses the typical assumption of linear approaches that the received signal is the result of a convolution with $h^{\prime}$. Indeed, the linearization of the initial equations implies that the resulting model will express the received pressure as a function of a convolution product with the PSF. It could also be expressed by taking $h^{\prime}$ out of the volume integral, ignoring the spatial aspect of the convolution (for $h^{\prime}$ ), and considering that $h^{\prime}$ is applied along the propagation axis (or ultrasonic line). This approximation is equivalent to saying that $h^{\prime}$ can be roughly viewed solely as a function of time. In this case, the time convolution makes it possible to move it at will inside the equation. It is also equivalent to assuming that the local variations of the medium properties are concomitant, so that the terms that are only a function of density can be expressed by its mean value. Following this point of view, consulting a table giving the speed of sound and density of different biological materials ${ }^{6}$ shows that the variations in the speed of sound in most cases imply a variation in density.

This assumption would imply in the volume integration that a term that is a function of the convolution of the gradients of the transmission and reception functions $h$ and $h^{\prime}$ is removed, which underlines the approximate aspect of such approaches and the importance of slow variations of $h$ and $h^{\prime}$ over the integration volume. It should also be noted that this is equivalent to Jensen's approximation, ${ }^{8}$ when he assumed that $h_{p e}$ is the solution of the homogeneous part of the wave equation, which consists in considering that $h$ can be replaced with $h_{p e}$ straightforwardly, discarding the additional terms that would appear when convolving the D'Alembert of $h$ by $h^{\prime}$.

\section{Expression of the established scattering coefficient}

Equations (37) and (38) clearly show the influence of the speed of sound $c$ on the scattering coefficient. A square dependence is obtained, in coherence with Fatemi and Kak's ${ }^{2}$ results. The expression of $R$ as a function of bulk modulus $K$ can also be linked to Gore and Leeman's ${ }^{1}$ result, and to the result of Zemp et al., ${ }^{7}$ which are partially a function of the local compressibility. Replacing $c$ and $\rho$ in Eq. (37) with Jensen's development [Ref. 8, Eq. (12)] expressed in Eq. (39) we have

$$
\begin{aligned}
& c=c_{0}+\Delta c, \\
& \rho=\rho_{0}+\Delta \rho
\end{aligned}
$$

with $\Delta c \ll c_{0}$ and $\Delta \rho \ll \rho_{0}$, and ignoring the higher-order terms gives

$$
R \approx \frac{\Delta \rho}{\rho_{0}}+\frac{2 \Delta c}{c_{0}},
$$

which is, except for the minus sign, Jensen's expression [Ref. 8, Eq. 47]

$$
R_{\mathrm{Jensen}}=\frac{\Delta \rho}{\rho_{0}}-\frac{2 \Delta c}{c_{0}} .
$$

The negative dependence of Jensen's scattering coefficient ${ }^{8}$ on the speed of sound variations $\Delta c$ does not seem to reflect a physical property of reflection because it implies that a positive variation of $c$ would reduce the local reflectivity or generate a negative scattering coefficient. Indeed, the nonlinear scattering coefficient for normal incidence $R_{\mathrm{NL}}=(Z$ $\left.-Z_{0}\right) /\left(Z+Z_{0}\right)$ shows that an increase in the local mechanical properties results in a positive scattering coefficient. Checking both developments suggests that a sign change is missing 
between Jensen's Eqs. (40) and (41) in Ref. 8. Fortunately, this sign mistake has no real impact on the extensive use of the simulator ${ }^{9}$ based on these equations, since Eq. (41) is not usually applied, the scattering coefficient $R_{\text {Jensen }}$ being directly defined instead. Finally, the comparison of Eqs. (40) and (41) suggests that $R_{\text {Jensen }}$ can be seen as a partial development of the expression of the scattering term $R$, and should be modified such that

$$
R_{\mathrm{Jensen}}^{+}=\frac{\Delta \rho}{\rho_{0}}+\frac{2 \Delta c}{c_{0}}
$$

is the corrected Jensen coefficient. ${ }^{8}$

It should be noticed that transforming the corrected Jensen coefficient ${ }^{8}$ would lead back to

$$
R_{\text {Jensen }}^{+}=\frac{\Delta \rho}{\rho_{0}}+\frac{2 \Delta c}{c_{0}}=\frac{c_{0}^{2} \cdot \Delta \rho+2 \cdot \rho_{0} \cdot c_{0} \cdot \Delta c}{\rho_{0} \cdot c_{0}^{2}}
$$

with the expression of $\rho_{0} \cdot c_{0}^{2}=K_{0}$ at the denominator underlining the closeness with the proposed model, while the numerator is like the first-order expression of $K$.

The expression of the scattering coefficient as function of the bulk modulus $K$ may be counterintuitive. On another hand, some assumptions such as the fact that the medium is considered as homogeneous until scattering occurs is also counterintuitive when considering imaging biological tissues, as well as the assumption that the waves are traveling at constant speed. The proposed model merely takes the typical set of assumptions close to their limits, showing their real impact on the expression of the scattering coefficient.

Figure 2 displays the values of the proposed scattering coefficient $R$ and the corrected Jensen coefficient $R_{\text {Jensen }}^{+}{ }^{8}$ Figure 2(a) illustrates the behavior of the scattering coefficients when the density is set at $\rho_{0}$ and Fig. 2(b) illustrates the behavior when the speed of sound is set at $c_{0}$. In this latter case, both coefficients are equal, but when density is set at $\rho_{0}$ [Fig. 2(a)], the $R$ and $R_{\text {Jensen }}^{+}$curves are tangential in $c_{0}$, showing their equivalence for small variations. However, $R$ remains greater than -1 when $c$ tends toward zero. The model presented in this paper is not quantitatively valid for large local variations $K$, but one can see in Eq. (37) that $R$ also tends to -1 as $K$ tends to zero and retains a physical meaning $(R>-1)$, which is advantageous for a linear approach of such cases. Unfortunately, the model suffers from its approximations for strong positive variations of $K$ above $K_{0}$, and Eq. (37) does not have any physical signification for $K>2 \cdot K_{0}$, slightly sooner than $R_{\text {Jensen }}^{+}$, with the scattering coefficient then greater than 1 . Nevertheless, the result given here provides an interesting approach for linearly modeling large local decreases in the mechanical properties of the tissue below their mean values. Those large decreases could reflect the existence of local very light inhomogeneities in the propagating medium, such as large bubbles or local vacuums.

For the simulation of such large variations, if needed, one should use the following definition to compute the scattering coefficient or to interpret the estimation results of local reflectivity:
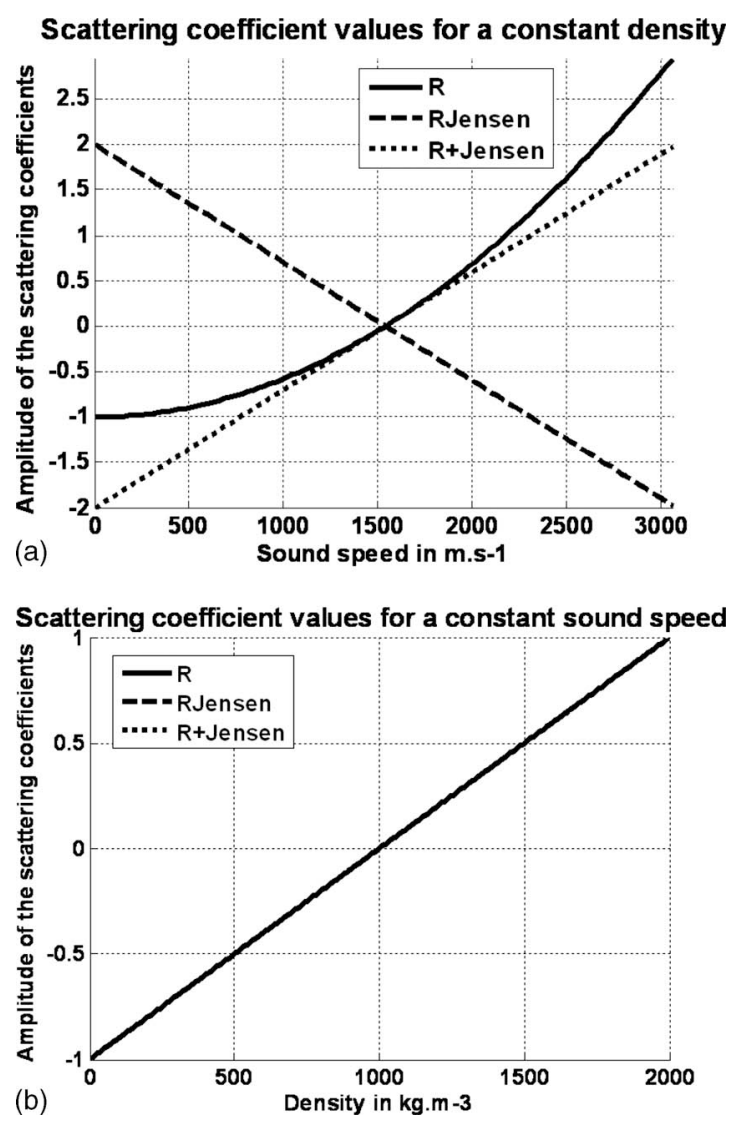

FIG. 2. Illustration of the dependence of the relative bulk modulus scattering coefficient $R$ on changes in speed of sound (a) and density (b) separately, compared to the values provided by the Jensen coefficient $R_{\text {Jensen }}$ (Ref. 8) and its modified version $R_{\text {Jensen }}^{+}$

$$
\begin{aligned}
& R=\frac{K-K_{0}}{K_{0}}, \quad K \in\left[0 ; 2 K_{0}\right], \\
& R=1, \quad K>2 K_{0} .
\end{aligned}
$$

Equation (44) underlines that, in the model proposed, when $K$ is greater than $2 \cdot K_{0}$, the solution of the inverse linear approach cannot distinguish dense matter and systematically underestimates the values of their mechanical properties. However, below $K_{0}$, the proposed model is coherent and more reliable [Fig. 2(a)] than Jensen's coefficient. ${ }^{8}$

Given that these two models-the one proposed by Jensen $^{8}$ and the one proposed in this paper-have very close, nearly equal expressions and given that their scattering coefficients, once corrected, are tangential around their mean value, they have the same ability to simulate the ultrasonic imaging process.

\section{CONCLUSION}

An expansion of the wave equation for propagation in an inhomogeneous medium has been established under the assumption of linear propagation. Solving it under the classical assumptions leads to an expression of the received rf signal that describes the local scattering coefficient as the variation of a single mechanical parameter. This model shows that considering the scattering as a function of small variations in density and speed of sound around their mean values is 
equivalent to associating the acoustical acquisition with a measure of the relative longitudinal bulk modulus. The model developed thus offers a way to estimate the bulk modulus of the imaged matter through the solution of the inverse problem in the case of small local variations. The final expression of the scattering coefficient is shown to be coherent with previous developments, while the model proposed is very close to the one presented by Jensen in 1991 (Ref. 8) and now widely used for ultrasonic imaging simulation. The similarity of the two models also implies that our model presents all the abilities of Jensen's model ${ }^{8}$ for simulating ultrasonic imaging and is more accurate when the scattering coefficient distribution is derived from the speed of sound and density fields. Moreover, the expression proposed is particularly convenient for the linear simulation of extremely soft inclusion imaging. The corresponding scattering coefficient, even if a linear model is intrinsically not well suited for such uses, retains a physical meaning when density or speed of sound decreases, even to zero.

${ }^{1}$ J. C. Gore and S. Leeman, "Ultrasonic backscattering from human tissue: A realistic model," Phys. Med. Biol. 22, 317-326 (1977).

${ }^{2}$ M. Fatemi and A. C. Kak, "Ultrasonic B-scan imaging: Theory of image formation and a technique for restoration," Ultrason. Imaging 2, 1-47 (1980).

${ }^{3}$ T. G. Leighton, The Acoustic Bubble (Academic, London, 1994), p. 100. ${ }^{4}$ R. T. Beyer and S. V. Letcher, Physical Ultrasonics (Academic, New York, 1969).

${ }^{5} \mathrm{~J}$. M. Mari, "Localisation échographique d'inclusions fortement échogènes en tissus mous, application à la détection d'électrode (Ultrasonic localization of strongly echogeneous inclusions in soft tissues, application to micro needle detection)," Ph.D. thesis, Université Claude Bernard Lyon 1,
France (2004).

${ }^{6}$ D. Royer and E. Dieulesaint, Ondes Élastiques Dans les Solides (Elastic Waves in Solids) (Masson, Paris, 1996), Vol. 1.

${ }^{7}$ R. Zemp, C. K. Abbey, and M. F. Insana, "Linear system models for ultrasonic imaging: Application to signal statistics," IEEE Trans. Ultrason. Ferroelectr. Freq. Control 50, 642-654 (2003).

${ }^{8} \mathrm{~J}$. A. Jensen, "A model for the propagation and scattering of ultrasound in tissue," J. Acoust. Soc. Am. 89, 182-190 (1991).

${ }^{9}$ J. A. Jensen, "Field: A program for simulating ultrasound systems," Med. Biol. Eng. Comput. 34, 351-354 (1996).

${ }^{10}$ J. A. Jensen, "Deconvolution of ultrasound images," Ultrason. Imaging, 14, 1-15 (1992).

${ }^{11}$ J. A. Jensen, D. Gandhi, and W. D. O'Brien, "Ultrasound fields in an attenuating medium," Proceedings of the IEEE 1993 Ultrasonics Symposium (1993), Vol. 2, pp. 943-946.

${ }^{12}$ M. E. Frijlink, D. E. Goertz, A. Bouakaz, and A. F. W. van der Steen, "Intravascular ultrasound tissue harmonic imaging: A simulation study," Ultrasonics, 44, e185-e188 (2006).

${ }^{13}$ F. Tranquart, N. Grenier, V. Eder, and L. Pourcelot, "Clinical use of ultrasound tissue harmonic imaging," Ultrasound Med. Biol. 25, 889-894 (1999).

${ }^{14}$ P. J. A. Frinking, A. Bouakaz, J. Kirkhorn, F. J. Ten Cate, and N. de Jong, "Ultrasound contrast imaging: Current and new potential methods," Ultrasound Med. Biol. 26, 965-975 (2000).

${ }^{15} \mathrm{M}$. Tang and R. Eckersley, "Frequency dependent attenuation and scattering by microbubbles," Ultrasound Med. Biol. 33, 164-168 (2007).

${ }^{16}$ M. Tang, R. Eckersley, and J. Noble, "Pressure-dependent attenuation with microbubbles at low mechanical index," Ultrasound Med. Biol. 31, 377384 (2005).

${ }^{17}$ J. M. Mari, K. Hibbs, and M. X. Tang, A Nonlinear Ultrasonic Scattering Approach for Micro Bubble Concentration Quantification (IEEE EMBC, Lyon, 2007).

${ }^{18}$ D. G. Duffy, Green's Function With Applications (Chapman and Hall/ CRC, Boca Raton, FL, 2001), pp. 99-101.

${ }^{19}$ D. Napolitano, C.-H. Chou, G. McLaughlin, T.-L. Ji, L. Mo, D. DeBusschere, and R. Steins, "Sound speed correction in ultrasound imaging," Ultrasonics 44, e43-e46 (2006). 